\title{
Urban Image Formation through Evaluation of Public Design Guideline of Bus stop in Jeju
}

\author{
SeoYoung Kim¹)
}

\begin{abstract}
The public space is very important in connecting the space and the bus stop is an important public place to maintain mutual exchanges. In order to improve the image of the city through the safe and convenient public transportation system, Jeju recently reorganized the public transportation system. As public transportation has improved, the use of public transportation has been revitalized, but the improvement in bus stops has been relatively low. In this study, investigating the bus stop in Jeju Island and divide the 'Public Design Guidelines' into two key words of three elements and examine the improvement direction. The results of the guideline evaluation showed that the safety and convenience functionalities were rated the highest, and the symbolic characteristics of the city 's characteristics and place characteristics were low. expecting that the bus stop in Jeju area will be the basic data that will help shape and improve the image of the city that will enhance the value as an international tourism city.
\end{abstract}

Keywords : Urban image formation, Evaluation of public design guideline, Bus stop, Public facilities

\section{Introduction}

\subsection{Purpose of Study}

Urban image is the core of city competitiveness in that it is the cultural and economic potential of the city to form the image of the city[1]. The public space of the city is a place where communities are communicated and various activities are exchanged. Bus stops provide the functional value of connecting urban space and space with cultural elements[2] are important public places to maintain mutual exchanges. The design of a bus stop in harmony with the city is an important element for forming the image of the city. Jeju is a region with diverse local and natural characteristics and needs a principle to form a city image. In order to improve the quality of life of residents, Jeju has been improving the public transportation in 30

Received(March 26, 2018), Review Result(1st: April 17, 2018, 2nd: May 8, 2018), Accepted(May 18, 2018)

${ }^{1}$ Dept. of Cultural Industry Mgmt., Honam University, 417, Eodeung-daero, Gwangsan-gu, Gwangju, Korea email: sykim@honam.ac.kr

* This paper was supported by Research Grant from Honam University in 2017 
years[3]. However, there is little change in the facility improvement factors of bus stops. The purpose of this study is to examine the facilities of the bus stop in Jeju Island and evaluate the basic criteria of 'Public Design Guideline' as three elements to check the improvement direction. By evaluating the basic elements of the design according to the guidelines for the design of public facilities in Jeju bus stop, which is suitable for a competitive international free city, it will be utilized in planning and improving the design of the bus stop facilities in the future.

\subsection{Research Scope and Method}

This paper extended the precedent paper of 'Evaluation of Public Design Guidelines for Bus Stop Jeju' by SeoYoung Kim (2017)[4]. The guideline evaluation criteria for bus stops were based on the 'Public Design Promotion Plan and Guidelines' (2017). Jeju is composed of administrative districts of Jeju City and Seogwipo City. Jeju City consists of four towns, two sides, and 19 provinces. Seogwipo City consists of three towns, two towns, and twelve towns. The scope of the survey was selected based on Jeju area (urban, central, mountainous \& urban, coastal), eup, myeon, and Dong bus stops. The evaluation method extracted two key words by elements based on the characteristics, function, and symbolism, which are the three basic elements of public design guidelines. In order to select the sample models of the bus stop, made a direct field survey and shooting. First, we classified them by region, and secondly by category, conducted guideline evaluation.

\section{Theoretical Background}

\subsection{Urban Image Formation}

The image formation of the city is based on the identity of the city[5]. The identity of the city is formed by the combination of the physical environment and cultural characteristics of the area. The physical elements of urban image formation include landscape and skyline, architecture aesthetics, urban architecture, public design, street trees, and urban environment colors. The physical environment element interacts with cultural features to form the image of the city. Efforts to form urban images are an important role that can become a competitive city and a city with a reputation. 


\subsection{Bus Stop}

A bus stop is a place where the bus stops for passengers getting on and off the bus, usually located in a walking space. In the form of a bus stop, only a sign or sign indicating that the bus stops at the location is provided, and a map showing the route or route through the stop is attached[6]. And many passengers with waiting passengers may develop into a waiting room with a roof, and if the route through the stop is very uncommon, there may be a timetable for a stop sign or a well visible place near the stop[6]. The bus stop is installed in the walking space at regular intervals, and it plays an important visual element to form the urban image. It is the most common public space for locals and visitors, and it is an important place to express local identity, cultural characteristics, and urban symbols. The facilities of the bus stop are classified into an illumination facility, a relaxation facility, a hygiene facility, a traffic facility, and an information facility[7]. The elements of bus stop in Jeju are classified as bus shelter, bench, trash can, bus stop sign, bus route map, and Bus Information Display Board.

\subsection{Public Design Guidelines Concept}

Jeju suggests a 'guideline' that defines the basic direction of public design as characterization, functionality, and symbolism in order to form a city 's unique identity[8].

(1) Characterization: It symbolizes the urban culture of Jeju Island, shows local characteristics, and provides a unified environment to establish the city 's history in the long run.

(2) Functionality: Functional design to improve the quality of life of the residents and improve the local service, all classes are easy to access and easy to use, and the safety of the residents is considered.

(3) Symbolism: The street facilities reflect the theme and place characteristics and meaning of the space to be installed and symbolize a pleasant, delayed friendly history, culture, and sightseeing city for the residents and visitors.

\section{Evaluation Model Classification}

There is no railway or subway on Jeju, and buses are the only public transportation. There are a total of 3,135 bus stops[9] in Jeju. A road connecting Jeju with an elliptical road along the coast and a middle mountain, and a road connecting North and South to the east and west, centered on Mt. Halla, are installed. In the evaluation model classification, extended the 
precedent paper of 'Evaluation of Public Design Guidelines for Bus Stop Jeju' by SeoYoung Kim (2017)[4]. For the evaluation of the public design guidelines of Jeju bus stop, a sample model was selected as a sample model for each of the six bus stops around Jeju (urban, central, mountainous \& urban, coastal). The sample model survey was divided into four times (January, June, September, and October in 2017), and the researcher directly took a sample model stop with the camera.

[Table 1] Sample Model

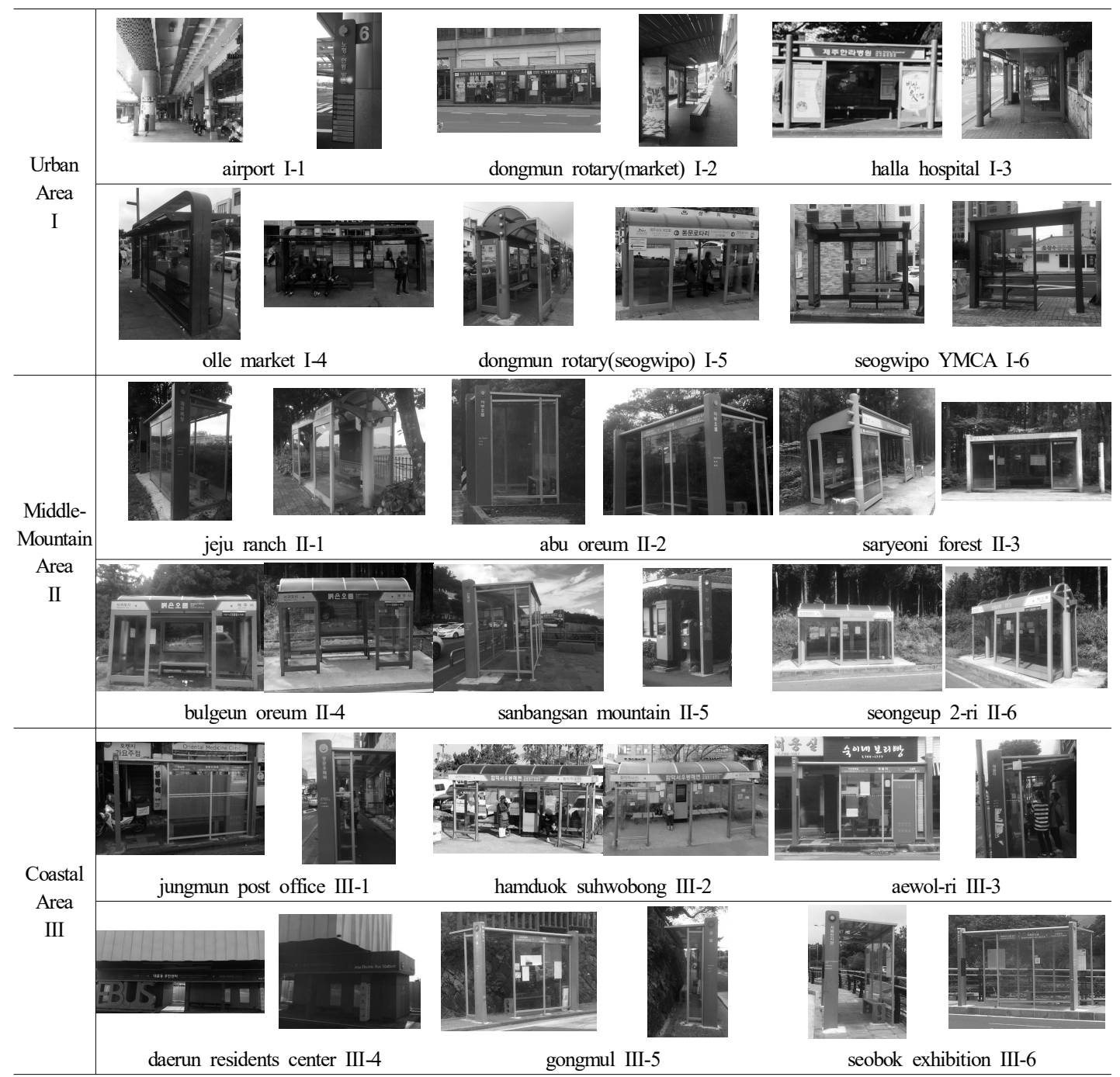




\section{Evaluation Model Analysis}

Based on the sample models, the facilities of bus stops were analyzed in depth. The types of bus shelters in Jeju bus stop are roof type and plate type. There are many roof types in urban areas and coastal areas, and many plate types in middle mountains. Bus shelters and benches have more than 10 different designs mixed together. The singularity is also a bench with heating on the bench. The bus stop sign is not installed independently, but there is a sign on the side post of the bus shelter or on the top of the front part. There are only three places in the downtown area where there are many passengers at the bus stop. Of the 3,135 bus stops[9], 1,200 bus information signboards (BIT) were installed[10][11], and bus information boards were not installed at bus stops in the middle mountain areas. There are also many bus stops of old facilities. Bus shelters and benches in urban areas and coastal areas have many designs that motivate the 'Jeongjusok' and 'Jeonggak',[12] the main forms of Jeju tradition. There are many bus shelters in Seogwipo City that express the excessive shape of local citrus fruits.

(1) Urban Area

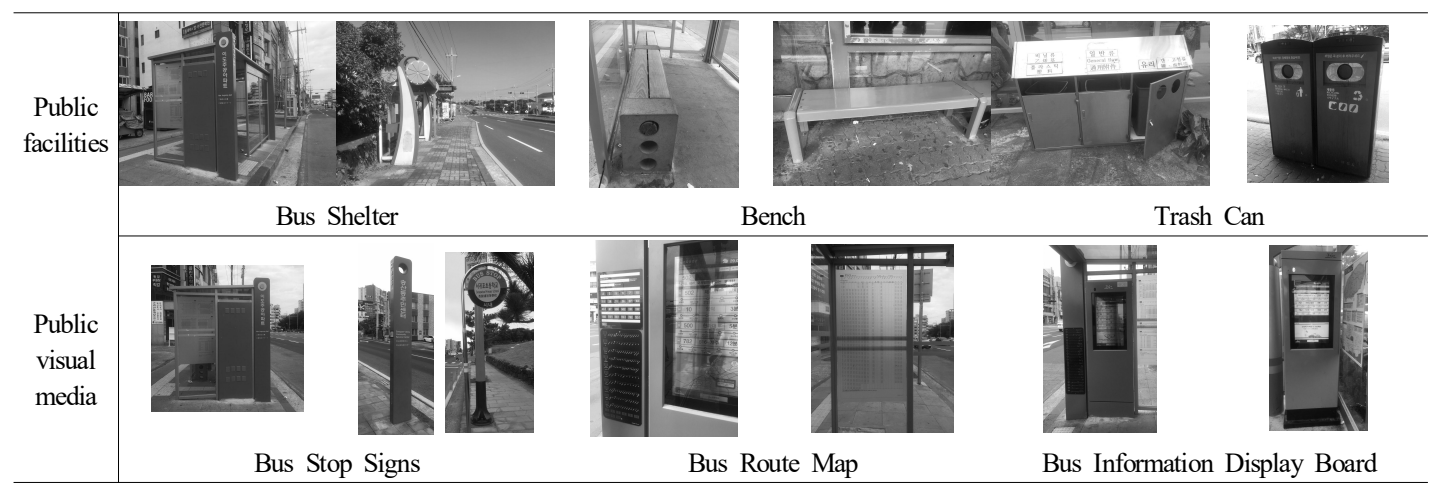

(2) Middle- Mountain Area

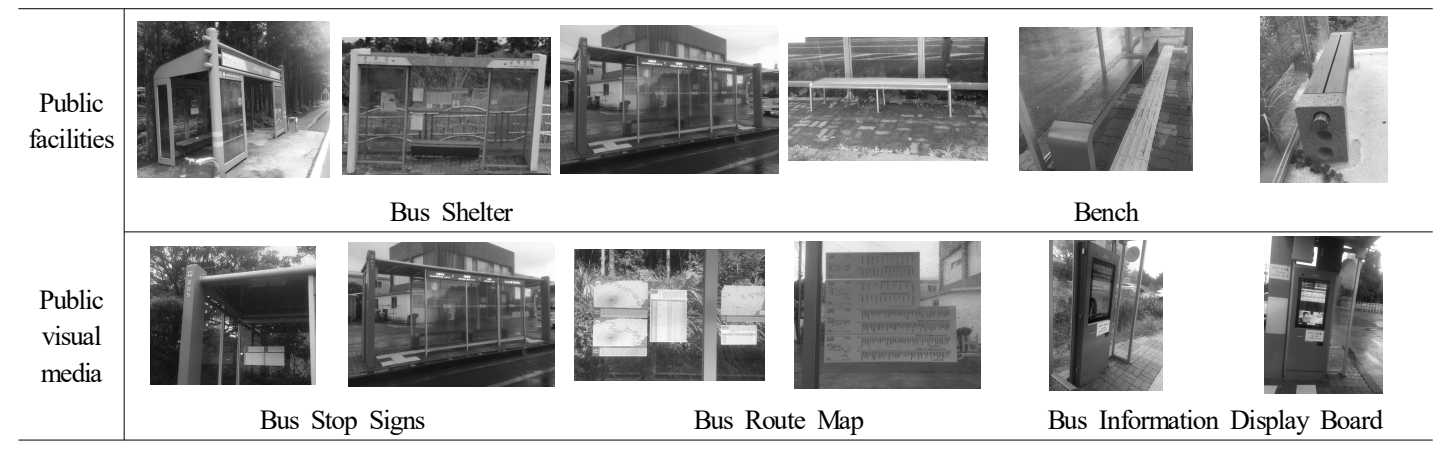

(3) Coastal Area 


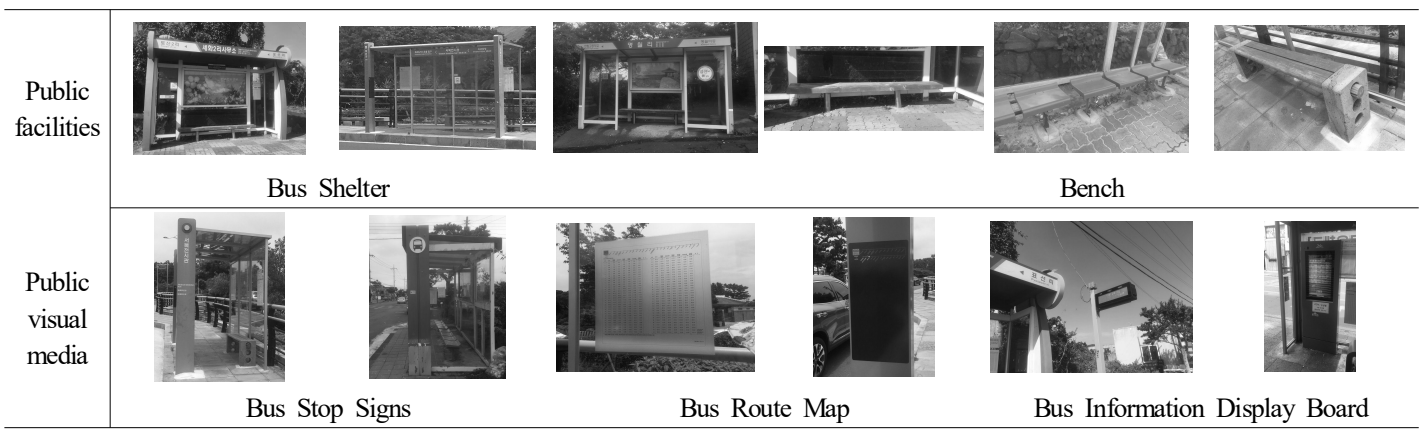

\section{Evaluation and Analysis}

\subsection{Evaluation of Public Design Guidelines}

The evaluation of the public design guidelines for bus stops was based on the three elements of the guidelines, characterization, functionality, and symbolism according to regional and public design facilities. Two key words were extracted from the three elements of the guideline and evaluated. Characterization is combine, local characteristics, functionality is convenient and safe, and symbolism is set as criteria of location feature and city symbol. The evaluators consisted of three design specialists and five untrained designers. The guideline scores were measured by the three step of the Likert scale[3][13]. The evaluation criteria score is 3 points for compliance, usually 2 points, and not for 1 point. [Table 2] shows the average scores of 8 evaluators.

[Table 2] Guideline Evaluation

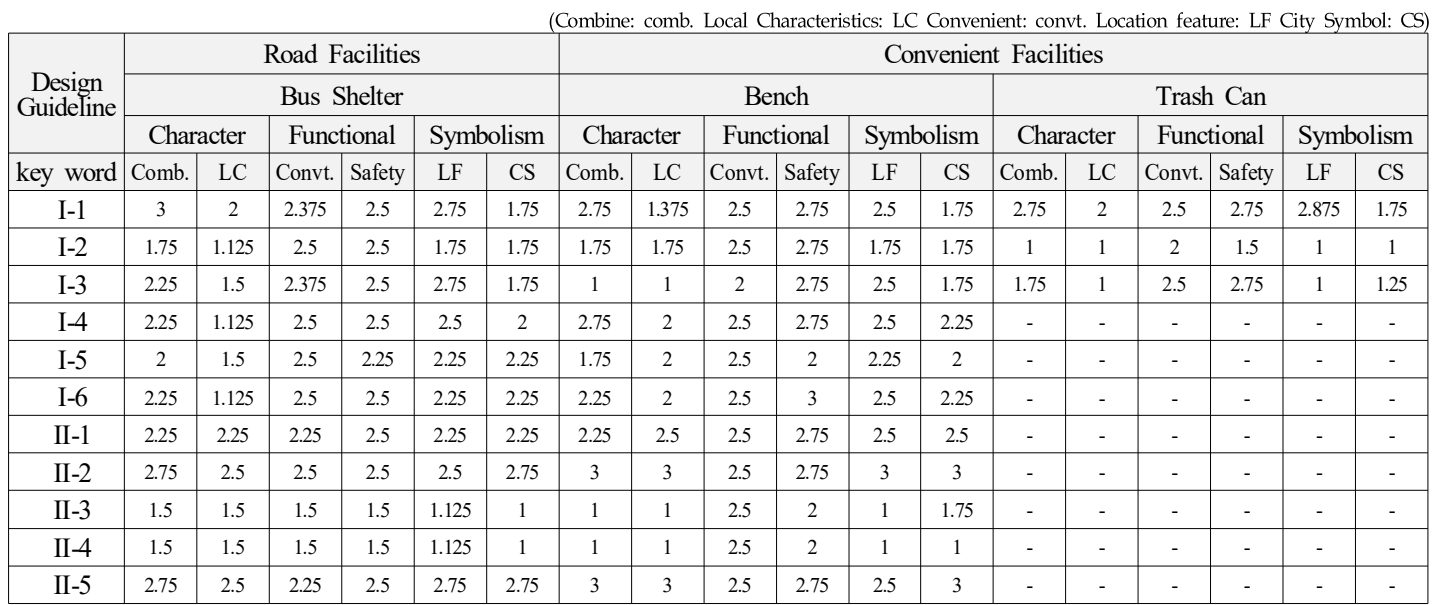




\begin{tabular}{|c|c|c|c|c|c|c|c|c|c|c|c|c|c|c|c|c|c|c|}
\hline II-6 & 2 & 2.25 & 2.25 & 1.5 & 2 & 2 & 1.75 & 1 & 2.5 & 2.25 & 1 & 2 & - & - & - & - & - & - \\
\hline III-1 & 2.75 & 2.5 & 2.5 & 2.5 & 2.5 & 2.75 & 3 & 3 & 2.5 & 3 & 2.75 & 3 & - & - & - & - & - & - \\
\hline III-2 & 1.5 & 1 & 2.25 & 1.5 & 1 & 1 & 1 & 1 & 2 & 2 & 1 & 1 & - & - & - & - & - & - \\
\hline III-3 & 2.75 & 2.5 & 2.5 & 2.5 & 2.5 & 2.75 & 1 & 3 & 2.5 & 3 & 2.75 & 3 & - & - & - & - & - & - \\
\hline III-4 & 2.75 & 2.5 & 2.5 & 2.5 & 3 & 3 & 3 & 2.75 & 2.5 & 2.75 & 3 & 3 & - & - & - & - & - & - \\
\hline III-5 & 2.75 & 2.5 & 2.5 & 2.5 & 2.5 & 2.75 & 3 & 3 & 2 & 3 & 2.75 & 3 & - & - & - & - & - & - \\
\hline III-6 & 2.75 & 2.5 & 2.5 & 2.5 & 2.5 & 2.75 & 3 & 3 & 2.5 & 3 & 2.75 & 3 & - & - & - & - & - & - \\
\hline \multirow{3}{*}{$\begin{array}{c}\text { Design } \\
\text { Guideline }\end{array}$} & \multicolumn{12}{|c|}{ Traffic Visual Media } & \multicolumn{6}{|c|}{ Visual Information } \\
\hline & \multicolumn{6}{|c|}{ Bus Stop Sign } & \multicolumn{6}{|c|}{ Bus Route Map } & \multicolumn{6}{|c|}{ Bus Information Display Board } \\
\hline & \multicolumn{2}{|c|}{ Character } & \multicolumn{2}{|c|}{ Functional } & \multicolumn{2}{|c|}{ Symbolism } & \multicolumn{2}{|c|}{ Character } & \multicolumn{2}{|c|}{ Functional } & \multicolumn{2}{|c|}{ Symbolism } & \multicolumn{2}{|c|}{ Character } & \multicolumn{2}{|c|}{ Functional } & \multicolumn{2}{|c|}{ Symbolism } \\
\hline key word & Comb. & LC & Convt. & Safety & LF & CS & Comb. & LC & Convt. & Safety & LF & $\mathrm{CS}$ & Comb. & LC & Convt. & Safety & LF & $\mathrm{CS}$ \\
\hline $\mathrm{I}-1$ & 3 & 3 & 3 & 2.75 & 3 & 3 & 2 & 2.5 & 2.875 & 3 & 2.75 & 2.75 & 2.5 & 2 & 2.25 & 2.25 & 1.375 & 2 \\
\hline $\mathrm{I}-2$ & 1.75 & 1.125 & 2.5 & 2.5 & 1.75 & 1.75 & 1.5 & 1.5 & 1.75 & 3 & 2.5 & 1.5 & 2.5 & 2 & 2.25 & 2.25 & 1.375 & 2 \\
\hline I-3 & 2.25 & 1.5 & 2.375 & 2.5 & 2.75 & 1.75 & 1.5 & 1.5 & 1.75 & 3 & 2.5 & 1.5 & 2.5 & 2 & 2.25 & 2.25 & 1.375 & 2 \\
\hline $\mathrm{I}-4$ & 2.25 & 1.125 & 2.5 & 2.5 & 2.5 & 2 & 1.5 & 1.5 & 1.75 & 3 & 2.5 & 1.5 & 2.5 & 2 & 2.25 & 2.25 & 1.375 & 2 \\
\hline I-5 & 2 & 1.5 & 2.5 & 2.25 & 2.25 & 2.25 & 1.5 & 1.5 & 1.75 & 3 & 2.5 & 1.5 & 2.5 & 2 & 2.25 & 2.25 & 1.375 & 2 \\
\hline I-6 & 2.25 & 1.125 & 2.5 & 2.5 & 2.25 & 2.25 & 2 & 1.5 & 1.75 & 3 & 2.5 & 2 & - & - & - & - & - & - \\
\hline II-1 & 2.25 & 2.25 & 2.25 & 2.5 & 2.25 & 2.25 & 1.5 & 1.5 & 1.75 & 3 & 2.5 & 2 & - & - & - & - & - & - \\
\hline III-2 & 3 & 3 & 3 & 3 & 3 & 2.75 & 3 & 3 & 3 & 3 & 2.5 & 2.75 & - & - & - & - & - & - \\
\hline II-3 & 1.5 & 2 & 1.5 & 1.5 & 1.125 & 1 & 1.5 & 1.5 & 1.75 & 3 & 2.5 & 1.5 & - & - & - & - & - & - \\
\hline II-4 & 1.5 & 1.5 & 1.5 & 1.5 & 1.125 & 1 & 1.5 & 1.5 & 1.75 & 3 & 2.5 & 1.5 & - & - & - & - & - & - \\
\hline II-5 & 2.75 & 2.5 & 2.25 & 2.5 & 2.75 & 3 & 3 & 3 & 3 & 3 & 2.5 & 2.75 & - & - & - & - & - & - \\
\hline III-6 & 1.75 & 2.25 & 2.25 & 1.5 & 2 & 2 & 1.5 & 1.5 & 1.75 & 3 & 2.5 & 1.5 & - & - & - & - & - & - \\
\hline III-1 & 3 & 3 & 3 & 3 & 3 & 3 & 3 & 3 & 3 & 3 & 2.5 & & 2.5 & 2 & 2.25 & 2.25 & 1.375 & 2 \\
\hline III-2 & 1.5 & 1 & 2.25 & 1.5 & 1 & 1 & 1.5 & 1.5 & 1.75 & 3 & 2.5 & 1.5 & 2.5 & 2 & 2.25 & 2.25 & 1.375 & 2 \\
\hline III-3 & 3 & 3 & 3 & 3 & 3 & 3 & 3 & 3 & 3 & 3 & 2.5 & 2.75 & 2.5 & 2 & 2.25 & 2.25 & 1.375 & 2 \\
\hline III-4 & 3 & 3 & 3 & 3 & 3 & 3 & 3 & 3 & 3 & 3 & 2.5 & 2.75 & 2.5 & 2 & 2.25 & 2.25 & 1.375 & 2 \\
\hline III-5 & 3 & 3 & 3 & 3 & 3 & 3 & 3 & 3 & 3 & 3 & 2.5 & 2.75 & - & - & - & - & - & - \\
\hline III-6 & 3 & 3 & 3 & 3 & 3 & 3 & 3 & 3 & 3 & 3 & 2.5 & 2.75 & - & - & - & - & - & - \\
\hline
\end{tabular}

\subsection{Analysis of Guideline Evaluation}

The evaluation results of the public design guidelines of bus stops in Jeju are as follows. In the guideline evaluation of the bus stop facilities, the bench was rated 2.3, the bus shelter 2.19, and the trash bin 1.8. The evaluation of guideline for bus route map was 2.37 points for bus route map, 2.36 points for bus stop sign, and 2.07 points for Bus Information Display Board. The bus stop sign was 2.52 points, which is the highest convenience in terms of functionality, and the trash can was rated at the lowest point of 1.33 points in terms of regional characteristics and symbolism in the characterization. 


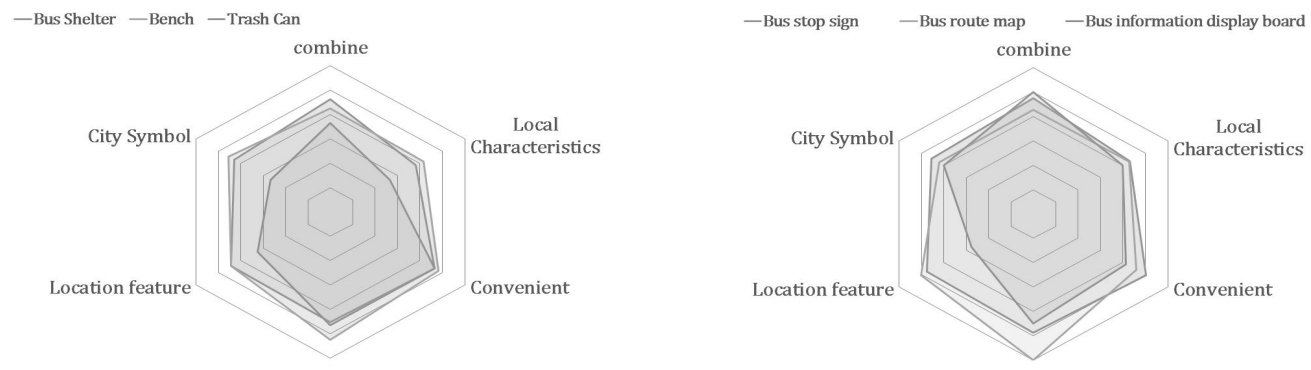

[Fig. 1] Guideline Evaluation Result

Among the six facilities, the local characteristics of the bus shelters were rated as 1.4. The trash cans were mostly bus stops that were not installed, and the surrounding areas were dirty because they could not be managed. In addition, there are a lot of high-gloss metal materials or street scenes that need to be improved and evaluated as the lowest point in all evaluations. The middle and coastal areas were rated as having the lowest symbolism. Among the six facilities, the place characteristics of the bench symbolism were evaluated as 1.83 . The primary color plastic bench stimulates the eyes and the use of materials and design that is not suitable for the place is a problem. Bus Information Display Board were standardized with the use of Jeju urban core color and accent color, and all areas were evaluated with the same scores in order of function, characterization, and symbolism.

[Table 3] Guideline Evaluation Result

\begin{tabular}{c|c|c|c}
\hline \multirow{2}{*}{ Area } & Road Facilities & Bench & Convenient Facilities \\
\cline { 2 - 4 } & Bus Shelter & Trash Can \\
\hline Urban Area & Functional $>$ Symbolism $>$ Character & Functional $>$ Symbolism $>$ Character & Functional $>$ Character $>$ Symbolism \\
\hline Middle-Mountain Area & Character $>$ Functional $>$ Symbolism & Functional $>$ Symbolism $>$ Character & - \\
\hline Coastal Area & Symbolism $>$ Functional $=$ Character & Symbolism $>$ Functional $>$ Character & - \\
\hline \multirow{2}{*}{ Area } & \multicolumn{2}{|c}{ Traffic Visual Media } & Public visual media \\
\hline Urban Area & Functional $>$ Symbolism $>$ Character & Functional $>$ Symbolism $>$ Character & Functional $>$ Character $>$ Symbolism \\
\hline Middle-Mountain Area & Character $>$ Functional $>$ Symbolism & Functional $>$ Symbolism $>$ Character & - \\
\hline Coastal Area & Functional $>$ Character $>$ Symbolism & Functional $>$ Character $>$ Symbolism & Functional $>$ Character $>$ Symbolism \\
\hline
\end{tabular}

\section{Conclusion}

The public space is very important in connecting the space and space of the city, and the bus stop is an important public place to maintain mutual exchanges. Jeju has recently 
reorganized public transportation in order to improve the quality of life of the citizens through forming a city image. As public transportation has improved, the use of public transportation has been revitalized, but the improvement in bus stops has been relatively low. In this paper, Jeju was classified into regional and institutional elements, and the three elements of 'Public Design Guidelines' were subdivided into two key words. The results of the guideline evaluation of this study are as follows. First, the evaluation results of the three elements of the bus stop are 2.42 for functionality, 2.081 for characterization, and 2.04 for symbolic. Functionality for ease of use and safety was the highest rating. However, local feature and the city symbols of symbolism were underestimated. It is required to improve the shape and color reflecting the characteristic of place for trash can and bus information signboard. Second, the evaluation results of the three elements of regional guidelines are 1.85 in urban area, 2.43 in functionality, and 1.99 in symbolism. The use of finishing materials that are not in harmony with the surrounding environment and the inconsistency between the facilities are lacking consistency and improvement is required. The characterization of the middle-mountain area was 2.06 points, the functionality 2.29 points, and the symbolism 2.06 points. It is necessary to improve the quality of the area because it uses a material that does not utilize the characteristics of the area and interferes with the continuity of the landscape by the mixed design. Characterization of the coastal area was 2.51, functionality was 2.58 , and symbolism was 2.38 . It is a form that does not take into consideration the walking space, abuses the city symbol of 'jeongnang', and tangerine. Third, the evaluation results of the 3 factors of the bus stop are as follows: Bus shelter 2.11, bench 2.11, trash bin 1.59, bus stop sign 2.27, bus guide 2.14, bus information signboard 2.27. The trash can needs to be improved in a generic form that does not match the surroundings and has no regional characteristics. Functionality is 2.29 for bus shelter, 2.53 for bench, 2.34 for trash, 2.48 for bus stop sign, 2.65 for bus sign, and 2.25 for bus information signboard. The bus shelter is required to be convenient to the user by ensuring stability and visual openness that is not subject to rain and wind damage[7]. Bus information display signboard is guiding to the location of the bus and the estimated time of arrival[10][11], which reduces convenience. Improvement of guidance information on traffic conditions is required. Symbolism is 2.18 for bus shelter, 2.25 for bench, 1.48 for recycle bin, 2.33 for bus stop sign, 2.31 for bus stop sign, and 1.69 for bus information signboard. Recycle bins are made from ready-made materials that do not match the place, so improvement is required. The purpose of this study is to examine the improvement directions of the above three factors through evaluating the guidelines of bus stop in Jeju area. It is expected that the bus stop in Jeju will be a basic data to form a valuable city image. 


\section{References}

[1] A. R. Han, and D. Y. Kwak, A Study on the Characteristics of the Media Facade Expression in Creating the Urban Image, Journal of the Korean Society of Design Culture (2012), Vol.18 No.3, pp.603-614.

[2] Park, Seok Hun, European City Wears Public Design: Germany's Landscape Facility Design, Ga-In Design Group (2007), pp.93

[3] https://ko.wikipedia.org, Likert Scale, Wikimedia Foundation, Dec. 18 (2017).

[4] S. Kim, Evaluation of Public Design Guidelines for Bus Stop Jeju, International Journal of ICT-aided Architecture and Civil Engineering (2017), Vol.4, No.2, pp.39-44.

[5] K. Lynch, The Image of City, The MIT Press, New York (1960), pp.8.

[6] https://ko.wikipedia.org, Bus Stop, Wikimedia Foundation, Jan. 5 (2018).

[7] City design of Jeju, Standard Design of Street Facilities, Jeju Special Self-Governing Province (2012).

[8] Design Architecture intellectual, Public Design Promotion Plans and Guidelines, Jeju Special Self-Governing Province (2017).

[9] http://www.jeju.go.kr/traffic, Jeju Special Self-Governing Province, Jan. 10 (2018).

[10] D. M. Go, Reorganization of Public Transport in Jeju Without 'Heavy Snow', http:/jeju.news1.kr/news/articleView.html?idxno=23751, Jan. 15 (2018).

[11] K. H. Kang, Jeju Public Transportation that Faces Heavy Snowfall, Jan. 16, Jimmy Daily (2018).

[12] K. Y. Kum, Hyundai Card, Jeju Island Bus Stop Design, March 14, Economic Review (2014).

[13] E. R. Babbie, The Practice of Social Research, Cengage Learning (2007), pp.238-239. 